03

\title{
Исследование реакции поверхности жидкости на импульсное воздействие наклонной газовой струи при малых числах Рэйнольдса
}

\author{
() А.П. Савенков, В.А. Сычёв \\ Тамбовский государственный технический университет, \\ 392000 Тамбов, Россия \\ e-mail: savencow@yandex.ru
}

Поступило в Редакцию 7 сентября 2021 г.

В окончательной редакции 31 октября 2021 г.

Принято к публикации 4 ноября 2021 г.

Получено математическое описание движения углубления на поверхности жидкости при наклонном действии газовой струи с использованием известных выражений для перемещения газового пузырька в жидкости. Определена граница преобладания силы вязкого трения над силой сопротивления формы. Взаимодействие газа и жидкости рассмотрено как динамический объект теории автоматического управления. Установлено, что динамические свойства двухфазной системы „струя газа - жидкость“ описываются уравнениями интегрирующего звена. При помощи специально созданной установки экспериментально получены переходные характеристики системы струя газа-жидкость для аэродинамических воздействий под углами 20 и $50^{\circ}$ к поверхностям жидкостей с вязкостями 0.71 и $26.1 \mathrm{~Pa} \cdot \mathrm{s}$ (число Рэйнольдса $\mathrm{Re}<2$ ). Результаты исследований необходимы для анализа бесконтактного аэродинамического метода измерений вязкости жидкостей.

Ключевые слова: бесконтактный, взаимодействие, вязкость, измерение, импульс, поверхность жидкости, струя газа.

DOI: 10.21883/JTF.2022.02.52010.251-21

\section{Введение}

Наиболее значимым применением процессов взаимодействия струи газа с жидкостью является металлургия [1-6]. Струя, действующая на поверхность расплава металла, играет ключевую роль в химических превращениях и перемешивании взаимодействующих веществ. Взаимодействие плазменных струй атмосферного давления с техническими и биологическими жидкостями применяется для изучения свойств холодной плазмы [7-11]. В оптике форма углубления, образованного газовой струей на поверхности жидкости, используется в качестве шаблона для изготовления асферических зеркал [12].

Процессы взаимодействия струи газа с поверхностью жидкости позволяют реализовать бесконтактные измерения физических свойств жидкостей: плотности, поверхностного натяжения и вязкости [13-17]. Одной из основных предпосылок для применения бесконтактных методов измерений свойств жидкостей является их высокая вязкость (более $1 \mathrm{~Pa} \cdot \mathrm{s})$. При реализации контактных методов вязкие жидкости создают значительные трудности и затраты времени при заполнении измерительных емкостей, удалении газовых пузырьков и очистке измерительных инструментов. Бесконтактные методы измерений, основанные на деформации поверхности контролируемой жидкости газовой струей, являются единственными, которые обеспечивают получение достоверной информации о свойствах вязких жидкостей $[15,17]$.
Известно сравнительно небольшое количество работ, посвященных изучению взаимодействия газовой струи с поверхностью вязкой жидкости $(\operatorname{Re}<1)$ [12,14-20]. Наклонное воздействие газовой струи на поверхность вязкой жидкости позволяет генерировать стабильные релаксационные колебания [21]. На поверхности невязкой жидкости $(\operatorname{Re}>100)$ колебания носят хаотический характер $[2,5,6,10,18,22]$. Частота колебаний на поверхности вязкой жидкости в значительной степени определяется вязкостью, и такая зависимость может быть положена в основу бесконтактного метода измерений этой величины. Однако более высокой точностью измерений и более широким диапазоном обладает импульсный метод [17].

При реализации бесконтактного импульсного аэрогидродинамического метода измерений вязкости на поверхность контролируемой жидкости воздействуют газовой струей с образованием углубления, а об измеряемой величине судят по времени достижения углублением заданной формы с момента начала струйного воздействия [17]. Аэродинамическое воздействие прекращают до начала релаксационных колебаний поверхности жидкости. Для определения вязкости используется апериодический переходной процесс в двухфазной системе струя газа-жидкость, вызванный изменением ее входного параметра - интенсивности аэродинамического воздействия.

Исследованию переходных процессов в системе „струя газа - жидкость“ посвящены работы $[19,20]$. В работе [19] исследована реакция поверхности жидкости 
на короткий аэродинамический импульс, после воздействия которого сила инерции формирует струю жидкости. В работе [20] представлены результаты численного моделирования переходного процесса, возникающего в системе „струя газа - жидкость“ при ступенчатом перпендикулярном аэродинамическом воздействии (вязкость жидкости $1 \mathrm{~Pa} \cdot \mathrm{s})$. Взаимодействие неперпендикулярной газовой струи с поверхностью жидкости исследовано в работах [3,23-26]. В этих публикациях использованы невязкие жидкости $(\operatorname{Re}>100)$.

Задача настоящей работы заключается в математическом описании динамических свойств двухфазной системы „струя газа - жидкость“ при наклонном аэродинамическом воздействии.

\section{1. Теоретический анализ}

Для исследования динамического взаимодействия многофазных систем часто используют численное моделирование происходящих процессов [22,24,26-28]. Такой подход позволяет изучить изменение модулей и направлений векторов скоростей потоков во всех точках модели рассматриваемой двухфазной системы, однако результаты численного моделирования, в свою очередь, требуют обобщения информации, полученной об исследуемой системе. Для анализа свойств системы необходимы упрощенные аппроксимирующие уравнения [29]. Существенные трудности для численного моделирования возникают из-за высокой вязкости (более $1 \mathrm{~Pa} \cdot \mathrm{s}$ ) одной из сред. Однако и в случае невязкой жидкости численное моделирование нередко приводит к получению неудовлетворительных результатов $[6,30]$.

Наши исследования базируются на подходах теории автоматического регулирования. В работе [29] определены основные динамические звенья и структурная схема системы „струя газа-жидкость“, в настоящей работе получены динамические характеристики этих звеньев. Для математического описания системы „струя газа - жидкость“ используются известные интегральные выражения для совокупности сил, действующих на всю область взаимодействия газа и жидкости на поверхности раздела фаз.

На рис. 1 представлены изображения профиля углубления на поверхности жидкости в различные моменты времени от начала подачи газовой струи с интервалом $0.4 \mathrm{~s}$. Изображения получены при помощи установки, описанной в работе [31]. Для получения струи использована трубка, в торце которой установлена диафрагма с отверстием истечения газа диаметром $d=0.89 \mathrm{~mm}$ и коэффициентом расхода $\mu=0.68$. Давление $P$ перед отверстием истечения в процессе наблюдения поддерживалось постоянным на уровне $5.5 \mathrm{kPa}$. Отверстие истечения струи расположено на расстоянии $H=10.3 \mathrm{~mm}$ над поверхностью жидкости. Струя действовала на поверхность жидкость под углом $\alpha=20^{\circ}$.

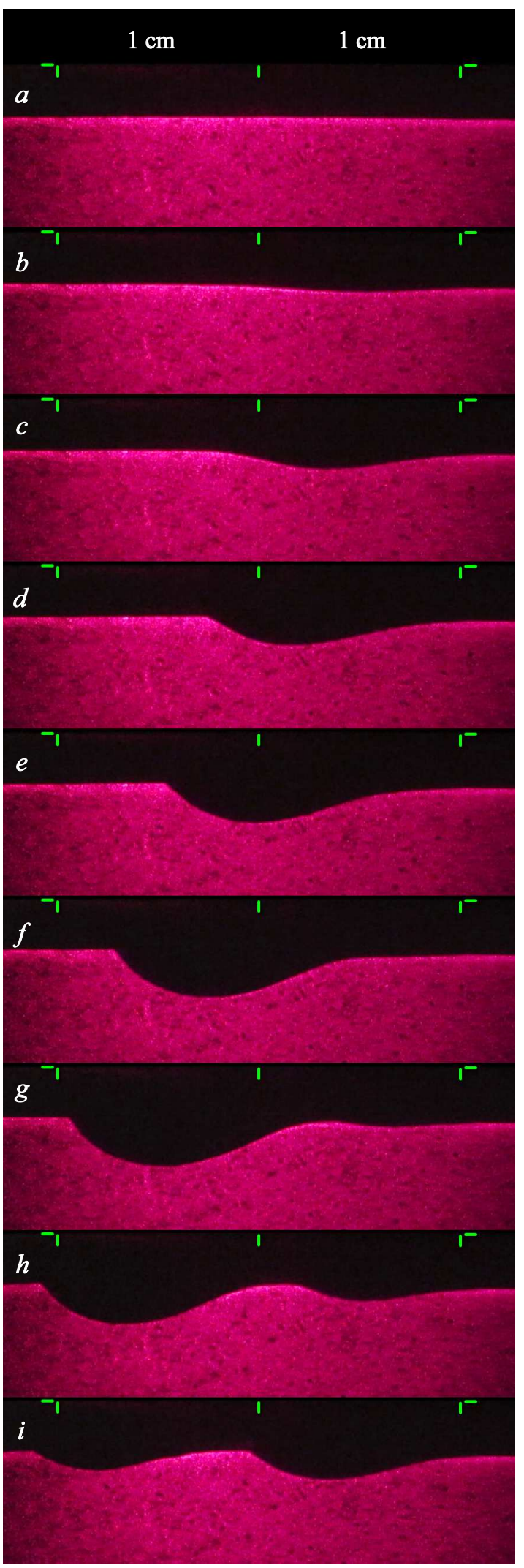

Рис. 1. Изображения профилей углубления на поверхности жидкости в различные моменты времени с начала подачи газовой струи: $a-i-$ соответствуют $0,0.4,0.8,1.2,1.6,2.0$, $2.4,2.8,3.2 \mathrm{~s}$ 


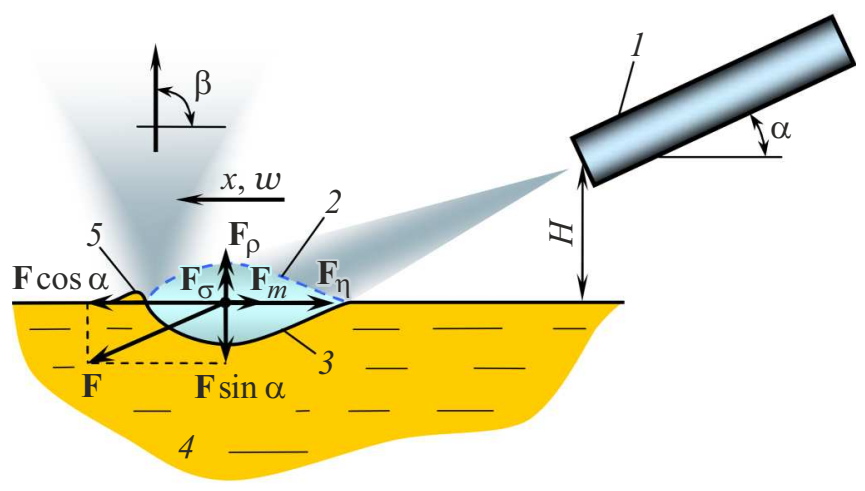

Рис. 2. Схема взаимодействия турбулентной газовой струи с поверхностью жидкости: 1 - струйная трубка с отверстием истечения струи, 2 - воображаемый газовый пузырек, 3 - углубление, 4 - жидкость, 5 - волна.

Жидкость - эпоксидная смола марки ЭД-20 производства компании „РоlyМах“ (Санкт-Петербург, Россия), динамическая вязкость $\eta=11.2 \mathrm{~Pa} \cdot \mathrm{s}$ при температуpe $27.0^{\circ} \mathrm{C}$.

Изображения, представленные на рис. $1, b-d$ иллюстрируют формирование углубления, рис. $1, d-h-$ его движение по поверхности жидкости в направлении действия газовой струи, рис. $1, g-i-$ зарождение нового углубления, уменьшение объема прежнего углубления и начало релаксационного колебательного процесса [21].

Для математического описания перемещения углубления по поверхности жидкости целесообразно использовать уравнение баланса сил на поверхности раздела фаз и известные формулы для движения газового пузырька в жидкости $[10,29,32,33]$. На воображаемый газовый пузырек 2 действуют следующие силы (рис. 2) [33]:

- $F$ газовой струи;

- $F_{\sigma}$ поверхностного натяжения;

- $F_{\rho}$ выталкивающая;

- $F_{\eta}$ вязкого трения;

- $F_{m}$ инерции.

При наклонном аэродинамическом воздействии основным выходным сигналом системы „струя газа - жидкость“ является горизонтальное перемещение $x$ углубления по поверхности жидкости. По времени достижения этой величиной заданного значения $x_{0}$ в бесконтактном аэрогидродинамическом методе измерения вязкости судят об измеряемой величине. Проекция сил на горизонтальную ось позволяет получить соответствующее уравнение баланса сил

$$
F_{m}+F_{\eta}=F \cos \alpha
$$

Уравнение (1) существенно упрощает процессы, происходящие на поверхности раздела фаз. В действительности вертикально направленные статические силы $F_{\sigma}$ и $F_{\rho}$ оказывают влияние на размер углубления, скорость его формирования и, как следствие, на коэффициент сопротивления и скорость перемещения. Однако, благодаря тому, что сила $F_{\sigma}$ меньше силы $F_{\rho}$ (см. работы $[15,29])$, а значения плотности большинства обычных жидкостей близки друг другу, уравнение (1) позволяет получить выражения для описания движения углубления с погрешностью в единицы процентов.

Сила действия струи на поверхность жидкости определяется по формуле [29]

$$
F=k F_{0}=k \frac{\pi d^{2} \mu}{2} P
$$

где $k-$ коэффициент формы углубления, $F_{0}-$ количество движения газа, вытекающее из струйной трубки 1 в единицу времени (рис. 2), [N]. Коэффициент $k$ зависит от изменения модуля и направления вектора количества движения газа при его взаимодействии с поверхностью жидкости. Из экспериментов известно, что газовые потоки выходят из углубления 3 под средним углом $\beta \approx 90^{\circ}$ к поверхности жидкости 4 независимо от угла $\alpha$ аэродинамического воздействия (в диапазоне $\alpha=15-75^{\circ}$, рис. 2). В этом случае направление количества движения газа в струе в результате взаимодействия с поверхностью жидкости изменяется на угол $90^{\circ}+\alpha$, а значение коэффициента формы теоретически определяется выражением $k=1+\sin \alpha$. В работе [29] получена соответствующая экспериментальная зависимость

$$
k=A+B \sin \alpha
$$

где $A=0.54, B=1.33$.

Учитывая (2) и (3), правую часть уравнения (1) можно переписать в виде

$$
F \cos \alpha=(A+B \sin \alpha) \cos \alpha F_{0} .
$$

Анализ выражения $(A+B \sin \alpha) \cos \alpha$ позволяет принять его постоянным и равным единице для диапазона углов $\alpha=20-50^{\circ}$.

Гидродинамическое сопротивление движению частицы в жидкости определяется по формуле [33]

$$
F_{d}=C_{d} \pi R^{2} \frac{\rho w^{2}}{2},
$$

где $C_{d}-$ коэффициент сопротивления; $R-$ радиус пузырька, $[\mathrm{m}] ; w-$ скорость его движения, $[\mathrm{m} / \mathrm{s}]$; $\rho$ - плотность жидкости, $\left[\mathrm{kg} / \mathrm{m}^{3}\right]$. При высокой вязкости жидкости и низкой скорости движения частицы $(\operatorname{Re}<1)$ преобладает действие силы вязкого трения $F_{\eta}$. При $\operatorname{Re}<0.01$ коэффициент сопротивления газовых пузырей определяется по формуле для твердых сферических тел [34]

$$
C_{d}=\frac{24}{\mathrm{Re}}
$$

a формула (5) преобразуется в формулу Стокса

$$
F_{\eta}=6 \pi R \eta w .
$$


С ростом числа Рэйнольдса наблюдается деформация пузырька, на коэффициент сопротивления оказывает влияние циркуляция газа внутри него. При $\mathrm{Re} \approx 1$ коэффициент сопротивления для газовых пузырьков определяется по формуле [34]

$$
C_{d}=\frac{16}{\mathrm{Re}} .
$$

Направление потоков газа в углублении противоположно направлению циркуляции газа в пузырьке (рис. 2), что приводит к увеличению коэффициента сопротивления. Форма углубления на поверхности жидкости также отличается от формы пузырька, поэтому в формуле (6) будем использовать коэффициент $b_{\eta}$, значение которого определим по данным эксперимента:

$$
F_{\eta}=b_{\eta} \pi R \eta w .
$$

При значениях числа Рэйнольдса, значительно превышающих единицу, вклад силы $F_{\eta}$ в силу сопротивления жидкости пренебрежимо мал, а коэффициент сопротивления стремится к единице [33]. Строго говоря, в диапазоне чисел Рэйнольдса от 1 до 100 возможны значения $C_{d}$ как большие, так и меньшие единицы, однако исключительно для оценки границы преобладания силы вязкого трения примем $C_{d}=1$ для $\operatorname{Re}>10[33,34]$. Для этих условий из формулы (5) получим значение силы сопротивления формы, обусловленной инерцией жидкости при обтекании углубления:

$$
F_{d}=\pi R^{2} \frac{\rho w^{2}}{2} .
$$

В настоящей работе рассматривается только случай преобладания вязкого трения, так как взаимодействие струи газа с невязкой жидкостью $(\operatorname{Re}>100)$ имеет хаотический характер, сопровождается уносом брызг и колебаний поверхности с разными частотами и длинами волн. Также очевидно, что измерение вязкости бесконтактным аэрогидродинамическим методом возможно только при преобладании силы вязкого трения. Исходя из условия $F_{\eta}>F_{d}$ из формул (6) и (8) получим максимальное допустимое значение числа Рэйнольдса

$$
\operatorname{Re}=\frac{2 R w \rho}{\eta}<24 .
$$

После подстановки скорости $w$ из формулы (6) в условие (9) получим условие преобладания силы вязкого трения над силой сопротивления формы в зависимости от вязкости жидкости -

$$
\eta>\sqrt{\frac{F_{\eta} \rho}{72 \pi}} \approx 0.066 \sqrt{F_{\eta} \rho} .
$$

Для оценки граничного значения вязкости в формуле (10) можно использовать силу $F$ действия газовой струи, так как сила $F_{\eta}$ вязкого трения является основной противодействующей ей силой. Изображения на рис. 1 получены в экспериментальных условиях, при которых сила $F$ составляла $\sim 4 \mathrm{mN}$. Для такого значения силы из выражения (10) получим граничное значение вязкости $\eta \approx 0.13 \mathrm{~Pa} \cdot \mathrm{s}$. При меньших значениях вязкости сила инерции $F_{m}$ в уравнении (1) должна включать член, отвечающий за действие силы сопротивления формы.

При вязкости жидкости, превышающей граничное значение $0.13 \mathrm{~Pa} \cdot \mathrm{s}$, сила инерции $F_{m}$ определяется ускорением присоединенной массы углубления. Для сферического газового пузырька радиусом $R$ сила инерции может быть оценена по формуле [33]:

$$
F_{m}=\frac{2}{3} \pi R^{3} \rho \frac{d^{2} x}{d t^{2}}
$$

где $t-$ время, [s]. Для учета асферичности углубления на поверхности жидкости и прочих факторов в формуле (11) вместо значения $2 / 3$ в дальнейшем будем использовать коэффициент $b_{m}$.

Контакт с жидкостью 4 имеет только половина поверхности пузырька 2 (рис. 2). При подстановке сил $F_{\eta}$ и $F_{m}$ в уравнение (1) выражения (7) и (11) целесообразно поделить на 2. С учетом выражения (4) из уравнения баланса сил (1) получим дифференциальное уравнение

$$
\frac{1}{2} b_{m} \pi R^{3} \rho \frac{d^{2} x}{d t^{2}}+\frac{1}{2} b_{\eta} \pi R \eta \frac{d x}{d t}=F_{0} .
$$

Начальными условиями для решения этого уравнения являются

$$
x(0)=x^{0}, \quad x^{\prime}(0)=0,
$$

где $x^{0}-$ позиция углубления в момент его формирования, $[\mathrm{m}]$.

После преобразования Лапласа из уравнения (12) получим передаточную функцию

$$
W(s)=\frac{x(s)}{F_{0}(s)}=\frac{2}{b_{m} \pi R^{3} \rho s^{2}+b_{\eta} \pi R \eta s},
$$

где $s-$ параметр преобразования Лапласа, $\left[\mathbf{s}^{-1}\right]$, и приведем ее к общепринятой форме

$$
W(s)=\frac{K}{s(T s+1)},
$$

где $K=2 /\left(b_{\eta} \pi R \eta\right)-$ коэффициент передачи, $[\mathrm{s} / \mathrm{kg}]$; $T=b_{m} R^{2} \rho /\left(b_{\eta} \eta\right)$ - постоянная времени, [s].

Аналогично работе [29] представим результаты теоретического анализа двухфазной системы „струя газа - жидкость“ при наклонном аэродинамическом воздействии в виде структурной схемы (рис. 3).

Потенциальная энергия сжатого газа (избыточное давление $P$ ) преобразуется элементом формирования струи (ЭФС) в кинетическую энергию струи с количеством движения $F_{0}$ в единицу времени и диаметром $d_{0}<d$. На рис. 3 для звена ЭФС показан коэффициент передачи по каналу $P-F_{0}$. Газовая струя взаимодействует с атмосферным воздухом $(A)$, в результате чего по 


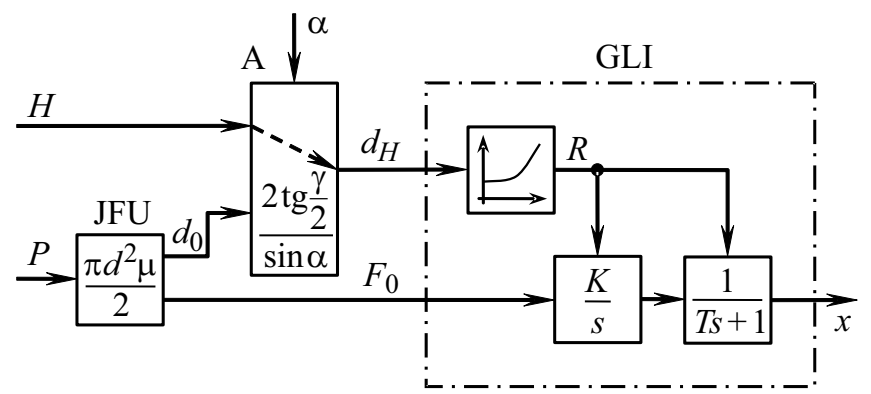

Рис. 3. Структурная схема двухфазной системы „струя газа - жидкость“ при наклонном аэродинамическом воздействии: JFU - Jet Forming Unit, элемент формирования струи (ЭФС); A - Atmosphere, атмосфера; GLI - Gas-Liquid Interface, поверхность раздела фаз (ПРФ).

мере удаления от ЭФС происходит линейное увеличение ее диаметра до значения $d_{H}$ у поверхности жидкости. Количество движения $F_{0}$ не изменяется.

Диаметр $d_{0}$ крайне слабо влияет на диаметр $d_{H}$ в связи с тем, что расстояние $H$ значительно превышает $d_{0}$ [35]. Для звена $A$ показан коэффициент передачи по каналу $H-d_{H}$. Угол $\gamma$ раствора конуса турбулентных струй, вытекающих из отверстия в тонкой стенке, слабо зависит от числа Рэйнольдса. В настоящее время угол $\gamma$, как правило, определяют для конуса, на поверхности которого скорость движения газа равна половине осевой. По данным работ $[35,36]$ значение этого угла составляет $5^{\circ}$. При постоянном расстоянии $H$ угол $\alpha$ наклона ЭФС влияет на длину свободного течения газа до поверхности раздела фаз (ПРФ), а, следовательно, и на диаметр $d_{H}$.

Дифференциальное уравнение (12) и передаточная функция (14) описывают динамические характеристики объекта ПРФ, который представлен в виде последовательного соединения интегрирующего и апериодического звеньев. Интегрирующее звено с передаточной функцией $K / s$ соответствует противодействию силы $F_{\eta}$ вязкого трения перемещению углубления, апериодическое силы $F_{m}$ инерции присоединенной массы углубления в момент подачи газовой струи до достижения постоянной скорости движения. Радиус $R$ углубления оказывает влияние на работу этих динамических звеньев, так как входит в формулы для определения их параметров $K$ и $T$. Связь радиуса $R$ с диаметром $d_{H}$ представлена в виде абстрактного нелинейного звена, так как на текущий момент неизвестно физически обоснованной математической зависимости, адекватно описывающей эту связь. При малых значениях $d_{H}$ радиус $R$ не обращается в нуль благодаря действию силы $F_{\sigma}$ поверхностного натяжения. Значения радиуса $R$ в конкретных условиях определяются экспериментальным путем.

Интегрирующее звено является астатическим. Согласно представленному математическому описанию и структурной схеме системы „струя газа-жидкость“, углубление будет двигаться на бесконечно большое рас- стояние до тех пор, пока действует газовая струя. В действительности большому перемещению углубления препятствует внутренняя обратная связь, под действием которой формируется новое углубление и исчезает старое. Наблюдается релаксационный колебательный процесс. Механизм колебательного режима взаимодействия струи газа с поверхностью жидкости подробно описан в работе [21]. Изучение обратных связей в системе „струя газа - жидкость“ выходит за рамки настоящей работы, так как при реализации рассматриваемого импульсного аэрогидродинамического метода измерений вязкости струйное воздействие прекращают до наступления колебательного процесса. На структурной схеме (рис. 3) соответствующие обратные связи не показаны.

После подстановки формулы (2) в уравнение (12) получим дифференциальное уравнение системы „струя газа - жидкость“ по каналу $P-x$ :

$$
\frac{b_{m} R^{3} \rho}{\mu d^{2}} \frac{d^{2} x}{d t^{2}}+\frac{b_{\eta} R \eta}{\mu d^{2}} \frac{d x}{d t}=P
$$

с начальными условиями (13). Решение уравнения (15) позволяет определить переходную характеристику системы „струя газа-жидкость“, как реакцию на ступенчатое пневматическое воздействие вида $P \cdot 1(t)$, где $1(t)$ - функция Хевисайда. Уравнение переходной характеристики имеет вид

$x(t)=x^{0}+\frac{b_{m} R \rho \mu d^{2} P}{b_{\eta}^{2} \eta^{2}}\left[\exp \left(-\frac{b_{\eta} \eta}{b_{m} R^{2} \rho} t\right)-1\right]+\frac{\mu d^{2} P}{b_{\eta} R \eta} t$.

В этой функции первое слагаемое определяется точкой отсчета перемещения $x$ углубления, второе отвечает за действие силы $F_{m}$ инерции, а третье за действие силы $F_{\eta}$ вязкого трения. Оценим значимость экспоненты в функции (16). Зададим $b_{\eta}=6, b_{m}=2 / 3$, $\rho=1000 \mathrm{~kg} / \mathrm{m}^{3}$ и используем для граничного значения вязкости $\eta=0.13 \mathrm{~Pa} \cdot \mathrm{s}$ (см. формулу (10)) данные, известные из эксперимента: радиус $R=5 \mathrm{~mm}$ и время деформации $t=0.1 \mathrm{~s}$. Получим значение экспоненты, равным 0.009. Для значения $\eta=0.3 \mathrm{~Pa} \cdot \mathrm{s}$ значение экспоненты составляет менее $10^{-4}$, что значительно меньше единицы. Следовательно, в изучаемом диапазоне вязкости выражение, стоящее в функции (16) в квадратных скобках, можно принять равным минус единице. Из функции (16) получим линейную переходную характеристику

$$
x(t)=x^{0}-\frac{b_{m} R \rho \mu d^{2} P}{b_{\eta}^{2} \eta^{2}}+\frac{\mu d^{2} P}{b_{\eta} R \eta} t .
$$

При вязкости $2 \mathrm{~Pa} \cdot \mathrm{s}$ второе слагаемое функции (17) принимает значение $0.068 \mathrm{~mm}$ и с увеличением вязкости стремительно падает (для расчета использованы значения величин, указанные выше). Это позволяет для вязких жидкостей представить переходную характеристику в упрощенном виде

$$
x(t)=x^{0}+\frac{\mu d^{2} P}{b_{\eta} R \eta} t .
$$




\section{2. Экспериментальная часть}

Для экспериментальной проверки выполненных теоретических исследований и определения действительных значений коэффициентов $b_{m}$ и $b_{\eta}$ использована установка, схема которой представлена на рис. 4. Над поверхностью жидкости 11 , находящейся в сосуде 8 , размещена планка 2 , на которой закреплена струйная трубка 1 с отверстием истечения диаметром $d=0.89 \mathrm{~mm}$ и коэффициентом расхода $\mu=0.68$, направляющая 3 и линейка 4 . Печатная плата лазерного триангуляционного детектора (TD) [37] поверхности жидкости может горизонтально перемещаться по направляющей 3. Для вертикального перемещения элементов 1-7 и TD используется привод D.

Сжатый воздух от линии пневматического питания подается в струйную трубку 1 через редукционный клапан Z типа РДВ5М, распределитель Y типа П1ПР.5 и ламинарный дроссель $\mathrm{R}$ (пневматическое сопротивление). Давление $P$ в струйной трубке регулируется при помощи клапана $\mathrm{Z}$ и контролируется по показаниям манометра М типа M-1/4 и калиброванного преобразователя давления PT типа MPX5010DP. Ресивер T предназначен для снижения пульсаций давления питания в моменты переключения распределителя Ү. Управление

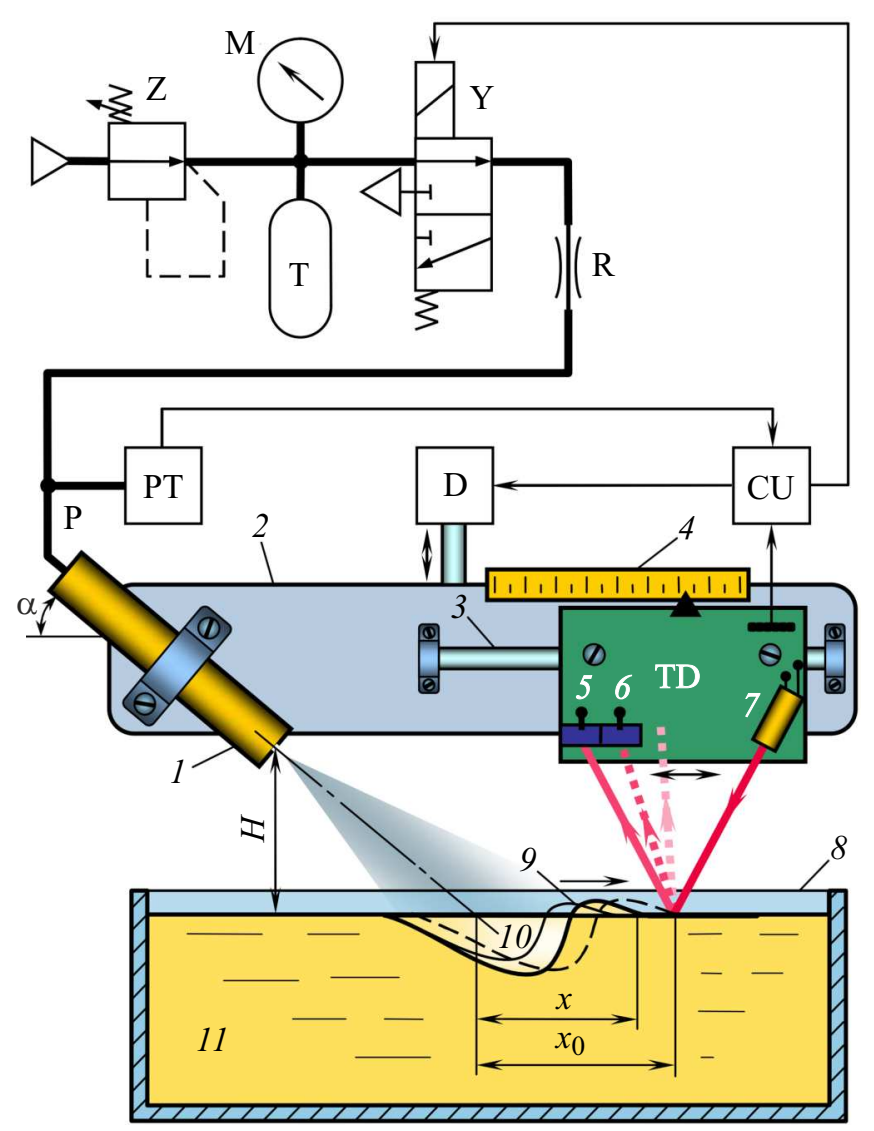

Рис. 4. Схема экспериментальной установки для исследования взаимодействия струи газа с поверхностью жидкости (объяснение обозначений на рисунке приведено в тексте). работой установки реализуется блоком управления $\mathrm{CU}$ на базе микроконтроллера ATmega16A.

TD предназначен для автоматической установки струйной трубки 1 на заданное расстояние $H$ относительно поверхности жидкости 11 и определения наличия ее деформации газовой струей в заданной точке. Подробное описание работы установки представлено в работе [17], TD - в работе [37].

Установка позволяет определять время достижения волной 9 заданного перемещения $x_{0}$. Перемещение $x$ волны 9 отсчитывается от точки пересечения оси газовой струи с плоскостью недеформированной поверхности жидкости 11 и до точки падения луча лазерного диода 7 на нее. При нажатии на соответствующую кнопку блока управления CU открывается распределитель Y, начинается счет времени $t$ действия струи и непрерывное измерение давления $P$ при помощи преобразователя РТ. Газовая струя, выходящая из струйной трубки 1 , формирует углубление 10 с волной 9. УГлубление перемещается в направлении действия струи и при достижении перемещения $x_{0}$ отклоняет отраженный луч лазерного диода 7. В момент времени, когда этот луч переходит с поверхности фотодиода 5 на поверхность фотодиода 6 , закрывается распределитель Y, завершается счет времени $t$ действия струи и определяется среднее за это время значение давления $P$. Полученные значения $t$ и $P$ выводятся на дисплей блока управления CU. При необходимости выполняется корректировка давления $P$ при помощи клапана Z.

Для экспериментального определения переходной характеристики $x(t)$ измерения времени $t$ действия газовой струи выполняются многократно при различных заданных значениях $x_{0}$ перемещения $x$. Изменение $x_{0}$ реализуется при помощи перемещения TD по горизонтальной направляющей 3 и контролируется по линейке 4 .

Эксперименты проведены с использованием касторового масла марки DAB-10 $(\eta=0.710 \mathrm{~Pa} \cdot \mathrm{s}$, $\rho=957 \mathrm{~kg} / \mathrm{m}^{3}$ ) производства AMEE CASTOR \& DERIVATIVES LTD (Банаскантха, Индия) и эпоксидной смолы марки ЭД-20 $\left(\eta=26.1 \mathrm{~Pa} \cdot \mathrm{s}, \quad \rho=1123 \mathrm{~kg} / \mathrm{m}^{3}\right)$ производства ООО НПК „Астат“ (г. Дзержинск, Россия) при температуре $25.0 \pm 0.1^{\circ} \mathrm{C}$ для значений угла $\alpha$, равных 20 и $50^{\circ}$. Вязкость определена при помощи калиброванного вискозиметра Брукфильда модели LVF, плотность - пикнометрическим методом. При $\alpha=20^{\circ}$ значение расстояния $H$ выбрано равным $10.3 \mathrm{~mm}$, при $\alpha=50^{\circ}-16.7 \mathrm{~mm}$.

\section{3. Результаты и их обсуждение}

На рис. 5 представлены экспериментальные переходные характеристики $x(t)$ системы „струя газа - жидкость“. Для сохранения положения графиков по оси абсцисс при различных значениях давления $P$ и 

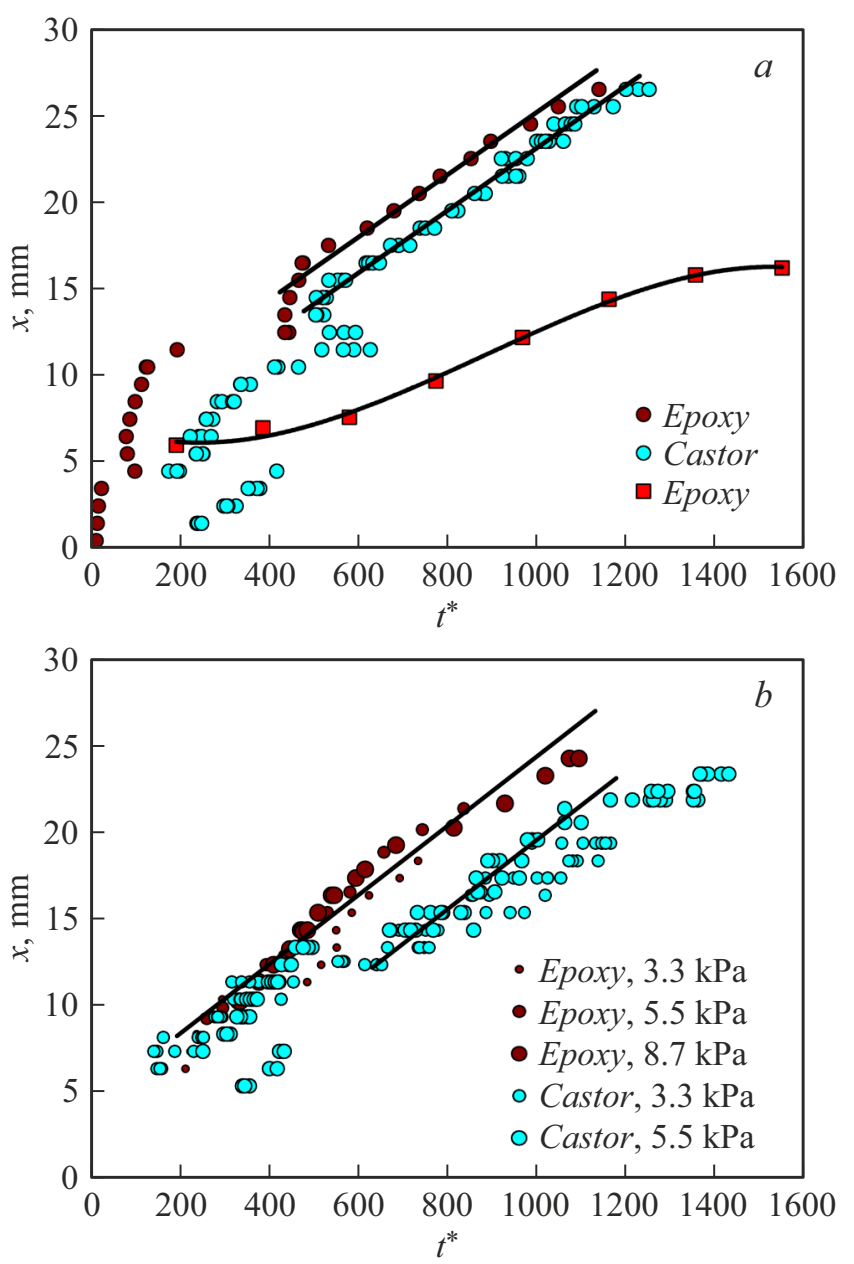

Рис. 5. Переходные характеристики системы „струя газа - жидкость“ для угла $\alpha=20(a)$ и $\alpha=50^{\circ}(b): \bullet-$ получены при помощи экспериментальной установки (рис. 4), получены в результате обработки изображений углубления (рис. 1), линии - результаты аппроксимации.

вязкости $\eta$ использован безразмерный параметр времени

$$
t^{*}=\frac{P}{\eta} t .
$$

Все зависимости, представленные на рис. 5, $a$, получены при давлении $P=5.5 \mathrm{kPa}$.

На рис. 5, $а$ для эпоксидной смолы марки ЭД-20 представлены данные, полученные при помощи экспериментальной установки (рис. 4) и по результатам анализа изображений углубления (рис. 1). Эти зависимости отличаются друг от друга размером волны 9 (рис. 4). По изображениям рис. 1 перемещение $x$ определяется по точке пересечения профиля углубления с недеформированной поверхностью жидкости. Установка (рис. 4) позволяет определять $x$ по фронту волны 9. Все аппроксимирующие линии при $t=0$ сходятся к расстоянию $x \approx 6 \mathrm{~mm}$, что соответствует радиусу углубления в начале деформации поверхности жидкости.
Экспериментальные зависимости, полученные при помощи установки (рис. 4), имеют характерные разрывы. Это обусловлено тем, что при $x=0-6 \mathrm{~mm}$ луч TD падает на поверхность формирующегося углубления 10 , а при $x=6-15 \mathrm{~mm}$ - в различные точки поверхности формирующейся волны 9 (рис. 4). Данные, отражающие перемещение углубления по поверхности жидкости, соответствуют диапазону $x=15-27 \mathrm{~mm}$. Для данных представленных на рис. $5, b$, такой диапазон соответствует $x=14-25 \mathrm{~mm}$. За верхними пределами этих диапазонов скорость движения углубления уменьшается, так как часть количества движения газа в струе затрачивается на формирование нового углубления (рис. 1).

Экспериментальные данные, полученные при помощи установки (рис. 4), аппроксимированы функцией (18), данные, полученные по изображениям рис. 1, - произвольным полиномом третьей степени. Данные для касторового масла при $\alpha=50^{\circ}$ и $P=3.3 \mathrm{kPa}$ не аппроксимировались в связи с их значительным отличием от остальных. Все данные для эпоксидной смолы при $\alpha=50^{\circ}$ аппроксимированы одной функцией.

Во всех аппроксимирующих функциях (18) использовано теоретическое значение коэффициента $b_{\eta}=6$. В качестве радиуса $R$ использована половина максимального размера поперечного сечения углубления в плоскости недеформированной поверхности жидкости. Значения радиуса $R$ определены по изображениям углублений в вертикальной плоскости перпендикулярной плоскости, в которой получены изображения, представленные на рис. 1. Для углов $\alpha$, равных 20 и 50॰ , получены значения радиуса 5.0 и $4.5 \mathrm{~mm}$ соответственно. В результате аппроксимации определялся единственный параметр $x^{0}$.

В таблице представлены результаты аппроксимации переходных характеристик системы „струя газа-жидкость, функцией (18). Значения $x^{0}$ для касторового масла меньше, чем для эпоксидной смолы. Исходя из предположения, что разность этих значений равна второму слагаемому в функции (17), определены значения коэффициента $b_{m}$. Для углов $\alpha$, равных 20 и $50^{\circ}$, получены значения $b_{m}$, равные 3.3 и $6.6 \mathrm{~mm}$ соответственно, при теоретическом значении 0.67 (см. формулу (11)). Очевидно, что такое превышение теоретического значения коэффициента $b_{m}$ может лишь отчасти быть объяснено отклонением формы углубления от сферической (рис. 2). Наиболее вероятной причиной смещения переходных характеристик для касторового масла влево является переходной процесс в установке при открытии распределителя Y (рис. 4). Давление перед отверстием истечения нарастает по экспоненциальному закону (апериодическое звено первого порядка) с постоянной времени $\sim 50 \mathrm{~ms}$. Эксперимент проводится так, что средние за период действия струи значения давления равны указанным выше (3.3 и $5.5 \mathrm{kPa})$, однако начало аэродинамического воздействия оказывается значительно более слабым, чем его завершение.

Другой возможной причиной увеличения коэффициента $b_{m}$ относительно теоретического значения является 
Результаты аппроксимации переходных характеристик системы струя газа-жидкость функцией (18)

\begin{tabular}{|c|c|c|c|c|c|c|c|}
\hline$\alpha$ & $\eta, \mathrm{Pa} \cdot \mathrm{s}$ & $P, \mathrm{kPa}$ & Диапазон, mm & $x^{0}, \mathrm{~mm}$ & $\Delta x, \mathrm{~mm}$ & $\sigma x, \mathrm{~mm}$ & $\delta x, \%$ \\
\hline \multirow{2}{*}{$20^{\circ}$} & 0.71 & 5.45 & $14-26$ & 5.1 & 0.9 & 0.4 & 2.0 \\
\hline & 26.1 & 5.45 & $14-27$ & 7.2 & 1.2 & 0.6 & 2.8 \\
\hline \multirow[b]{2}{*}{$50^{\circ}$} & 0.71 & 5.45 & $14-21$ & -0.4 & 2.4 & 0.9 & 5.2 \\
\hline & 26.1 & $\begin{array}{l}3.27 \\
5.45 \\
8.71\end{array}$ & $\begin{array}{r}14-18 \\
9-21 \\
10-24\end{array}$ & 4.4 & 2.0 & 0.9 & 5.5 \\
\hline
\end{tabular}

Примечание: $\Delta x$ - максимальное абсолютное отклонение экспериментальных данных от функции (18); $\sigma x-$ среднее квадратическое отклонение экспериментальных данных; $\delta x$ - относительная погрешность аппроксимации.

противоположное направление циркуляции газа в углублении, формируемом газовой струей на поверхности жидкости, относительно направления циркуляции газа в поднимающемся газовом пузырьке. Движение газа вдоль границы раздела фаз в углублении вызывает более сильное течение жидкости в направлении перемещения углубления, чем течение жидкости вокруг поднимающегося газового пузырька того же объема.

Несмотря на несоответствие экспериментального значения коэффициента $b_{m}$ теоретическому, результаты математического описания движения углубления следует признать удовлетворительными. При изменении вязкости в 37 раз и плотности в 1.2 раза отклонение значений $x$ для разных жидкостей друг от друга в среднем составляет $10 \%$ при $\alpha=20$ и $30 \%$ при $\alpha=50^{\circ}$.

Взаимодействие наклонной газовой струи с поверхностью жидкости лучше соответствует теоретическому анализу при малых углах наклона $\left(20^{\circ}\right)$. Это проявляется в большем диапазоне линейного перемещения углубления и меньшей погрешности аппроксимации.

\section{Заключение}

Исходя из результатов теоретических и экспериментальных исследований переходных характеристики двухфазной системы „струя газа - жидкость“, можно сделать следующие выводы.

Для математического описания перемещения углубления по поверхности жидкости с успехом могут быть использованы известные выражения для сил, действующих на движущийся газовый пузырек. При этом коэффициент в формуле Стокса следует брать равным теоретическому значению 6 . Для исследования зависимости этого коэффициента от разных факторов целесообразно использовать установку, в которой в момент определения триангуляционным детектором наличия деформации реализуется перемещение элементов устройства параллельно поверхности жидкости в направлении действия газовой струи.

Граничное значение вязкости, определяющее преобладание действия силы вязкого трения над силой сопротивления формы, зависит только от силы действия газовой струи на поверхность жидкости и ее плотности. В рассмотренных условиях граничное значение вязкости составило $0.13 \mathrm{~Pa} \cdot \mathrm{s}(\mathrm{Re}=24)$.

Система „струя газа - жидкость“ описывается уравнениями интегрирующего звена, что подтверждается наличием линейных участков на графиках переходных характеристик. Наилучшее соответствие теории наблюдается при малых углах аэродинамического воздействия.

Полученные уравнения переходных характеристик системы „струя газа - жидкость“ могут быть использованы для определения нижнего предела, модели и функции измерений вязкости жидкостей бесконтактным аэрогидродинамическим методом. Использованные математические зависимости и подходы к их получению позволяют проводить дальнейшие исследования двухфазной системы „струя газа - жидкость“, в частности, в режиме стабильных релаксационных колебаний.

\section{Конфликт интересов}

Авторы заявляют, что у них нет конфликта интересов.

\section{Список литературы}

[1] N. Dyussekenov, S.S. Park, H.Y. Sohn. Miner. Process. Extr. M., 120 (1), 21 (2011). DOI: $10.1179 / 037195510 X 12791826058136$

[2] J. Maruyama, K. Ito, M. Ando, J. Okada, K. Ito. ISIJ Int., 60 (6), 1375 (2020). DOI: 10.2355/isijinternational.ISIJINT2019-653

[3] Y. Chen, A.K. Silaen, C.Q. Zhou. Processes, 8 (6), 700 (2020). DOI: $10.3390 /$ pr8060700

[4] G.Q. Liu, G.X. Zhang, K. Liu. Metalurgija, 59 (3), 299 (2020).

[5] S. Sabah, G. Brooks. Ironmaking \& Steelmaking, 43 (6), 473 (2016). DOI: 10.1080/03019233.2015.1113755

[6] D. Muñoz-Esparza, J.-M. Buchlin, K. Myrillas, R. Berger. Appl. Math. Model., 36 (6), 2687 (2012).

DOI: 10.1016/j.apm.2011.09.052

[7] C.J. Ojiako, R. Cimpeanu, H.C.H. Bandulasena, R. Smith, D. Tseluiko. J. Fluid Mech., 905, A18 (2020). DOI: $10.1017 / \mathrm{jfm} .2020 .751$ 
[8] Б.Б. Балданов, А.П. Семенов, Ц.В. Ранжуров, Э.О. Николаев, С.В. Гомбоева. ЖТФ, $85(11), 156$ (2015). [B.B. Baldanov, A.P. Semenov, Ts.V. Ranzhurov, E.O. Nikolaev, S.V. Gomboeva. Tech. Phys., 60 (11), 1729 (2015). DOI: 10.1134/S1063784215110043]

[9] A. Stancampiano, E. Simoncelli, M. Boselli, V. Colombo, M. Gherardi. Plasma Sourc. Sci. Tech., 27 (12), 125002 (2018). DOI: 10.1088/1361-6595/aae9d0

[10] S. Park, W. Choe, H. Lee, J.Y. Park, J. Kim, S.Y. Moon, U. Cvelbar. Nature, 592, 49 (2021).

DOI: $10.1038 / \mathrm{s} 41586-021-03359-9$

[11] T.R. Brubaker, K. Ishikawa, H. Kondo, T. Tsutsumi, H. Hashizume, H. Tanaka, S.D. Knecht, S.G. Bilén, M. Hori. J. Phys. D: Appl. Phys., 52 (7), 075203 (2018). DOI: $10.1088 / 1361-6463 /$ aaf460

[12] W. Fu, X. Zhang. Optik, 207, 164451 (2020). DOI: $10.1016 /$ j.ijleo.2020.164451

[13] Д.М. Мордасов, М.М. Мордасов. ЖТФ, 87 (3), 468 (2017). DOI: $10.21883 /$ JTF.2017.03.44257.1910 [D.M. Mordasov, M.M. Mordasov. Tech. Phys., $62(3), \quad 490$ (2017). DOI: $10.1134 / \mathrm{S} 1063784217030148]$

[14] A.H. Pfund, E.W. Greenfield. Ind. Eng. Chem., 8(2), 81 (1936). DOI: 10.1021/ac50100a001

[15] М.М. Мордасов, А.П. Савенков, М.Э. Сафонова, В.А. Сычёв. Измерительная техника, (6), 55 (2018). DOI: 10.32446/0368-1025it-2018-6-55-60 [M.M. Mordasov, A.P. Savenkov, M.E. Safonova, V.A. Sychev. Meas. Tech., 61 (6), 613 (2018). DOI: 10.1007/s11018-018-1473-7]

[16] Б.В. Дерягин, В.В. Карасев. Успехи химии, 57 (7), 1110 (1988). DOI: 10.1070/RC1988v057n07ABEH003379 [B.V. Deryagin, V.V. Karasev, Russ. Chem. Rev., 57 (7), 634 (1988). DOI: 10.1070/RC1988v057n07ABEH003379]

[17] А.П. Савенков, М.М. Мордасов, В.А. Сычёв. Измерительная техника, (9), 43 (2020). DOI: 10.32446/0368-1025it.2020-9-43-49 [A.P. Savenkov, M.M. Mordasov, V.A. Sychev. Meas. Tech., 63 (9), 722 (2020). DOI: 10.1007/s11018-021-01845-0]

[18] A. He, A. Belmonte. Phys. Fluid., 22 (4), 042103 (2010). DOI: $10.1063 / 1.3327209$

[19] É. Ghabache, T. Séon, A. Antkowiak. J. Fluid. Mech., 761, 206 (2014). DOI: 10.1017/jfm.2014.629

[20] A. Balabel. Emirates J. Engineer. Res., 12 (3), 35 (2007).

[21] М.М. Мордасов, А.П. Савенков. Письма в ЖТФ, 42 (18), 25 (2016). [M.M. Mordasov, A.P. Savenkov. Tech. Phys. Lett., 42 (9), 940 (2016). DOI: 10.1134/S1063785016090224]

[22] M. Adib, M.A. Ehteram, H.B. Tabrizi. Appl. Math. Model., 62, 510 (2018). DOI: 10.1016/j.apm.2018.05.031

[23] R.D. Collins, H. Lubanska. Brit. J. Appl. Phys., 5(1), 22 (1954). DOI: $10.1088 / 0508-3443 / 5 / 1 / 306$

[24] J. Solórzano-López, R. Zenit, M.A. Ramírez-Argáez. Appl. Math. Model., 35 (10), 4991 (2011).

DOI: 10.1016/j.apm.2011.04.012

[25] O. McRae, A. Gaillard, J.C. Bird. Phys. Rev. E, 96 (1), 013112 (2017). DOI: 10.1103/PhysRevE.96.013112

[26] X.-T. Wu, R. Zhu, G.-S. Wei, K. Dong. J. Min. Metall. B, 56 (3), 307 (2020). DOI: 10.2298/JMMB190225019W

[27] R.B. Kalifa, S.B. Hamza, N.M. Saïd, H. Bournot. Int. J. Mech. Sci., 165, 105220 (2020). DOI: 10.1016/j.ijmecsci.2019.105220

[28] X. Zhou, Q. Yue, Z. Di, D. Sheng, M. Ersson. JOM, 73 (10), 2953 (2021). DOI: 10.1007/s11837-021-04810-y
[29] М.М. Мордасов, А.П. Савенков, К.Е. Чечетов. ЖТФ, 86 (5), 20 (2016). [M.M. Mordasov, A.P. Savenkov, K.E. Chechetov. Tech. Phys., 61 (5), 659 (2016). DOI: $10.1134 / \mathrm{S} 1063784216050170]$

[30] В.Н. Петров, А.С. Шабалин, В.Ф. Сопин, С.В. Петров, С.Л. Малышев. Вестн. технолог. ун-та, 20 (2), 85 (2017).

[31] М.М. Мордасов, А.П. Савенков. Измерительная техника, (7), 47, (2015). [M.M. Mordasov, A.P. Savenkov. Meas. Tech., 58 (7), 796 (2015). DOI: 10.1007/s11018-015-0796-x]

[32] V.A. Makarov, F.A. Korolev, R.E. Tyutyaev. IOP Conf. Ser.: Mater. Sci. Eng., 1047, 012014 (2021). DOI: $10.1088 / 1757-899 X / 1047 / 1 / 012014$

[33] P. Snabre, F. Magnifotcham. Eur. Phys. J. B Condens. Matter., 4 (3), 369 (1998). DOI: 10.1007/s100510050392

[34] Г.И. Келбалиев. ТОХТ, 45 (3), 264 (2011). [G.I. Kelbaliyev. Theor. Found. Chem. Eng., 45 (3), 248 (2011). DOI: $10.1134 /$ S0040579511020084]

[35] W.R. Quinn. Eur. J. Mech. B/Fluids, 25 (3), 279 (2006). DOI: 10.1016/j.euromechflu.2005.10.002

[36] J. Mi, P. Kalt, G.J. Nathan, C.Y. Wong. Exp. Fluid., 42 (4), 625 (2007). DOI: $10.1007 / \mathrm{s} 00348-007-0271-9$

[37] М.М. Мордасов, А.П. Савенков, М.Э. Сафонова, B.A. Сычёв. Автометрия, $54(1), \quad 80 \quad$ (2018), DOI: 10.15372/AUT20180111 [M.M. Mordasov, A.P. Savenkov, M.E. Safonova, V.A. Sychev. Optoelectron., Instrum. Data Process., 54 (1), 69 (2018). DOI: $10.3103 / \mathrm{S} 8756699018010119]$ 